\title{
Long Run Cointegration In Asean-4 Stock Market: What We Learned?
}

\author{
Eddy Soegiarto $\mathrm{K}$. \\ Faculty of Economic, \\ Universitas 17 Agustus 1945 Samarinda, \\ Jl. Ir. H. Juanda No. 80, 75124, Indonesia \\ Faizal Reza \\ Faculty of Economic, \\ Universitas 17 Agustus 1945 Samarinda, \\ Jl. Ir. H. Juanda No. 80, 75124, Indonesia

\section{Adisthy Shabrina Nurqamarani} \\ Faculty of Economic, \\ Universitas 17 Agustus 1945 Samarinda, \\ Jl. Ir. H. Juanda No. 80, 75124, Indonesia
}

\begin{abstract}
ASEAN-4 is an emerging market that experienced promising developed financial sector in recent years. The interaction between stock prices and macroeconomic variables has been extensively researched by researchers. By using secondary monthly ASEAN-4 data from January 2009 to December 2017 and using the ARDL estimation, it was found that exchange rates and GDP had a long-term positive effect on stock prices. While inflation, current account, and GDP have no effect on stock price formation. It is also found that there are co-integration in the short and long term in four ASEAN-4 countries. To strengthen the results of co-integration, using "one to one" interactions concluded that inflation, current accounts and interest rates have a very small contribution to the share price in ASEAN-4.
\end{abstract}

Keywords: macroeconomic variables, stock price, ARDL, cointegration.

\section{INTRODUCTION}

The relationship between macroeconomic variables and stock prices has been extensively studied until now, various tests were conducted by researchers to find causality between macroeconomic variables and stock prices, one of the most popular theories is Arbitrage Pricing Theory (APT). Various findings suggest that the investigation surrounding this issue is far from satisfactory, the difficulty in determining the determinant factors affecting stock prices in APT approach brings its own challenges for researchers. Huberman and Wang (2005) suggest that based on economic intuition, researchers continously add new factors which are too numerous to mention in the relationship between stock prices and economic variables. Since there is no specific consensus of any determinant factor affecting stock prices, then its result many macroeconomic variables that can be checked in relation to the stock price itself. Even Reza et al. (2018) states that since there are changes in countries behavior in the trade sector, a definite relationship among the variables can not be held on this issue. ASEAN-4 as an emerging market that have potential land for investors to obtain a large yield, uncertainty in the global financial sector is currently characterized by a larger capital inflow to developing countries than any other region in the world. 
The ability of developing countries to create and sustain their economic growth also causes the financial sector in these countries to be seen as more secure and stable. For examples, the economic growth of India and Indonesia in recent years have been a magnet for investors to invest in developing countries. Based on some reports in the long term conditions in developing countries can be said positive. Overall contribution of developing countries to global economic growth in 2015-2016 amounted to 58.15\%. In 2017, the growth of Gross Domestic Product of Southeast Asia countries is quite encouraging where Indonesia reaching 5.1\%, Cambodia 6.9\%, Malaysia 5.9\%, Philippines and Myanmar 6.7\%, Vietnam 6.8\% Thailand and Singapore respectively 3.9\% and 3.6\%. Based on data from the IMF and Word Bank, during the period 1990-2017 the average growth of exporters of commodities such as Malaysia and Indonesia amounted to 5.3\%, while the growth of importers of commodities such as Thailand and the Philippines amounted to $4.8 \%$.

The good prospect in the emerging market financial sector is in consequence of the macroeconomic factors that have been maintained in recent years. Despite strong export performance and strong domestic demand, some major economies in Asia such as China, Indonesia, Malaysia and Vietnam are conducting fiscal consolidation efforts in 2018. Historically, the interaction between macroeconomic variables and the financial sector has been studied by experts for few decades. Zubair (2013) using Granger Causality states that there is no relationship between monetary indicators (exchange rates and M2) before and during Nigeria's financial crisis. Wickremansinghe (2016) using emerging market data tested the relationship between macroeconomic variables and stock price during 1985 to 2004. It was concluded that there are a long-term and short-run relationship between stock prices and macroeconomic variables in Sri Lanka. Belgacem (2013) uses the macroeconomic variable announcement on French stock prices, concluding that the importance of information about the United States economy on the development of the world economy.

Bahloul et. al. (2017) with a sample of developed and developing countries found that during 2002-2014 the index of Islamic stocks in developed and developing countries was influenced by money supply and conventional stock returns both on high volatility and low volatility regimes, while other macroeconomic variables concluded did not affect index of Islamic stocks at high volatility regime. Although there is few research about interaction of stock price and macroeconomic variables on emerging markets that have identical characters in one region in Asia, but it still yet to be found how macroeconomic variables work to influence stock prices in exact way. This paper aims to see whether there is a long-term relationship between macroeconomic variables and stock prices in ASEAN-4 countries. This will help us to understand the channel and how macroeconomic force influenced the stock price. The remainder of this paper will be presented as follows: section two is a literature review, section three provide research methodology, section four discuss the results and finally the section five is conclusions of the paper.

\section{LITERATURE REVIEW}

There is one big theory in asset pricing theory that underlies macroeconomic variable relationship and stock price namely Arbitrage Pricing Theory (APT). This theory states that the formation of stock price is not only influenced by single factor $\beta$ risk as what as Capital Asset Pricing Model (CAPM) told at the beginning of asset pricing model development. Although some experts argue that CAPM is more intuitive and theoretically easy to apply, however there are several reasons why APT is seen as having an advantage over CAPM. First, APT does not require assumption of distribution from asset return. Secondly, we no need the strong assumptions about utility like in the CAPM derivation. Third, with consider some risk sources 
in APT so that the forecast will be better than CAPM. Finally, there is no need for the special role of an efficient market portfolio as in the CAPM theory (Rasiah and Kim, 2011).

The formation of stock prices in APT can be said to be influenced by macroeconomic factors. Until now a lot of economic factors that have been analyzed by experts in relation to stock prices in various capital markets in the world. Some variables that are often associated with stock prices include exchange rates, inflation, interest rates, current accounts, GDP, oil prices, etc. Because APT considered many factors that affected stock market,it also seen asa weakness of APT that not considered have a good theoretical foundation on the habits of investors. Some experts believe that it is dangerous to focus only on mean-beta space as some studies show that the generating return asset process is also influenced by other factors such as Earning Price Ratio, firm size, etc. (Rasiah and Kim, 2011). First attempts to examine the interaction between macroeconomic variables and stock returns were performed by Chen, Roll, and Ross (1986) who found that stock returns in the US were also influenced by macroeconomic factors.

Further studies using macroeconomic variables show some empirical findings that vary from one another. Yau and Nieh (2009) with non-linear regression stated that there is a long-term relationship between the New Taiwan Dollar (NTD) exchange rate against Japanese Yen (JPY) with stock prices in Japan and Taiwan from January 1991 to March 2008. Their research also found that there is a long-term cointegration between the NTD / USD exchange rate against the stock price in Taiwan. Umoru and Asekome (2013) conclude that there is a bidirectional cointegration and causality between Naira's exchange rate against US Dollar and the stock price in Nigeria. These results support flow and stock-oriented economic theory. Sharma and Mahendru (2010) using weekly data found that there was an impact between exchange rates and gold prices on stock prices during January 2008 to January 2009 in India. Rahman and Uddin (2009) found no cointegration and causality among stock prices and exchange rates in the three South Asian countries namely Bangladesh, India and Pakistan.

While Parsva and Tang (2017) found a biderectional causality between exchange rates and stock prices in three of the four Middle East countries namely Iran, Oman and Saudi Arabia during January 2004 and December 2011, Quadir (2012) found that although there was a positive relationship between macroeconomic variables i.e treasury bill interest rate and production industrial sector on stock returns in Dhaka Exchange Rate during January 2000 to February 2007, but he concluded no significance among the variables studied. Patel (2012) states that in the long run there is a relationship between macroeconomic variables and stock indices in India from January 1991 to December 2011. Exchange rates, industrial production indices, inflation, money supply, and commodity prices such as gold, oil and silver is also an important factor in determining the stock index. Ouma and Muriu (2014) concluded that during 2003-2013 using APT and CAPM, money supply, exchange rates, and inflation affected stock returns in Kenya, while the interest rate does not affect the stock return. From many studies, a general conclusion can be drawn is from Pilinkus (2011) that stated investors need to pay attention to the different effects of macroeconomic variables on stock indices as a result of speculative motives. He stated that the relationship between stock index and macroeconomic variables is more reliable in the long run.

\section{METHODOLOGY}

In this paper we use monthly data of stock price and five macroeconomic variables i.e exchange rate, inflation, interest rate, current account, and GDP from January 2009 until December 2017. Stock price data accessed from investing.com, while for macroeconomic variables obtained from central banks and statistical authority of each country. To ensure 
validity data, we also check data from other sources such as from the World Bank, IMF, and Asian Development Bank (ADB) reports. To estimate the interaction between stock prices and macroeconomic variables in ASEAN-4 countries, we will follow the following equation:

$$
S P_{t}=\alpha_{0}+\alpha_{1} E x R_{t}+\alpha_{2} \operatorname{Inf} f_{t}+\alpha_{3} C u A_{t}+\alpha_{4} \operatorname{InRt}+\alpha_{5} G D P_{t}+e_{t} \ldots \ldots \ldots
$$

Where $\alpha_{0}$ is a constant, $\alpha_{\mathrm{i}}$ is the coefficient where $i=1,2, \ldots, \mathrm{n}$. SP is the stock price, ExR is the exchange rate of each country against US Dollar, while Inf is inflation, $\mathrm{CuA}$ is current account, InR and GDP is the interest rate and Gross Domestic Product, and $e_{t}$ is error term. The existence of different order of integration in variables that we estimate can be used by Autoregressive Distributed Lag Model (ARDL) which can we write in the following form:

$S P_{t}=\beta_{0}+\gamma_{0} E x R_{t}+\delta_{0} I n f_{t}+\varphi_{0} C u A_{t}+\phi_{0} I n R_{t}+\theta_{0} G D P_{t}+\sum_{i=0}^{n} \beta i S P t-i+\sum_{i=0}^{n} \gamma i E x R t-i+$ $\sum_{i=0}^{n} \delta i \operatorname{Inft}-i+\sum_{i=0}^{n} \varphi i C u A t-i+\sum_{i=0}^{n} \phi i I n R t-i+\sum_{i=0}^{n} \theta i G D P t-i+\varepsilon_{t}$

From equation (2), we can find for the cointegration of ARDL with following equation:

$$
\begin{aligned}
& \Delta S P_{t}=\Psi_{0}+\sum_{i=0}^{p} B i \Delta E x R t-i+\sum_{i=0}^{p} C i \Delta \operatorname{Inft}-i+\sum_{i=0}^{p} D i \Delta C u A t-i+\sum_{i=0}^{p} E i \Delta \operatorname{InR} t-i+ \\
& \sum_{i=0}^{p} F i \Delta G D P t-i+\rho_{1} S P_{t-1}+\sigma_{2} E x R_{t-1}+\tau_{3} \operatorname{Inf} f_{t-1}+\omega_{4} C u A_{t-1}+\Upsilon_{5} \operatorname{In} R_{t-1}+\psi_{6} G D P_{t-1}+\zeta_{t}
\end{aligned}
$$

Where $\Delta$ is the first difference operator, $B_{i}, C_{i}, D_{i}, E_{i}, F_{i}$ are variable coefficients, $p$ is lags length, and $\zeta_{t}$ is an error term. By using cointegration of ARDL, we can estimate the long run of the ARDL equation as follows:

$$
S P_{t}=\eta_{0}+\eta_{1} E x R+\eta_{2} \operatorname{Inf}+\eta_{3} C u A+\eta_{4} \operatorname{InR}+\eta_{5} G D P+\xi_{t}
$$

We use unrestricted Error Corection Model in this paper to see the dynamic short term parameters of the respective ARDL equations of ASEAN-4 countries by the formula:

$$
\begin{aligned}
& \Delta S P_{t}=\lambda_{0}+\sum_{i=0}^{p} \lambda 1 i \Delta S P t-i \sum_{i=0}^{p} \lambda 2 i \Delta E x R t-i+\sum_{i=0}^{p} \lambda 3 i \Delta \operatorname{Inft}-i+\sum_{i=0}^{p} \lambda 4 i \Delta C u A t-i+ \\
& \sum_{i=0}^{p} \lambda 5 i \Delta \operatorname{InR} t-i+\sum_{i=0}^{p} \lambda 6 i \Delta G D P t-i+\pi E C T_{t-1}+v_{t} \ldots \ldots \ldots . . .(5)
\end{aligned}
$$

Where $\pi$ is the coefficient of ECT showing dynamic short term cointegration and adjustment speed. ECT coefficients with negative and significant values indicate there is short-term cointegration in the equations that we estimate.

\section{Descriptive Statistics and Unit Root Test}

\section{FINDINGS AND DISCUSSION}

As in the estimation of time series equations, the interaction between stock prices and macroeconomic variables in this paper is done by testing the stationarity between variables. In this paper to sharpen the conclusions of the paper, we choose and examine the countries that categorized of emerging markets and developing economies based on the IMF classification, which means that the countries we examine have similar characteristics to each other. Differences in estimates will lead us to new perspectives on causality between macroeconomic variables and stock prices in emerging markets in some Southeast Asia countries. Table 1 is a descriptive statistic of ASEAN-4 countries. 
Table 1. ASEAN-4 Descriptive Statistics

\begin{tabular}{|c|c|c|c|c|}
\hline \multirow{2}{*}{ Variable } & \multicolumn{4}{|c|}{ Mean } \\
\hline & Philippines & Malaysia & Indonesia & Thailand \\
\hline Stock Price & 5709,024 & 1589,961 & 4248,688 & 1237,894 \\
\hline Exchange Rate & 45,3834 & 3,5177 & 11107,99 & 32,6949 \\
\hline Inflation & 3,0472 & 2,2733 & 0,4029 & 1,258 \\
\hline Current Account & 0,6063 & 4,4309 & $-3,1526$ & 4,2354 \\
\hline Interest Rate & 3,7939 & 2,8843 & 6,4468 & 2,6551 \\
\hline GDP & 167577 & 239902,5 & 19995 & 8872816 \\
\hline \multicolumn{5}{|c|}{ Standard Deviation } \\
\hline Stock Price & 1852,407 & 233,1736 & 1134,89 & 338,7687 \\
\hline Exchange Rate & 2,7088 & 0,473 & 1873,054 & 1,8968 \\
\hline Inflation & 1,4199 & 1,3435 & 0.5334 & 2,2409 \\
\hline Current Account & 0,5545 & 2,5605 & 3,7211 & 5,3438 \\
\hline Interest Rate & 0,4868 & 0,3813 & 1,0116 & 0,6833 \\
\hline GDP & 356921 & 33186,23 & 391073,8 & 757617,4 \\
\hline \multicolumn{5}{|c|}{ Max Value } \\
\hline Stock Price & 8558,42 & 1882,71 & 6355,65 & 1753,71 \\
\hline Exchange Rate & 51,4049 & 4,4599 & 14406,03 & 36,1347 \\
\hline Inflation & 7,2 & 4,921 & 3,29 & 4,292 \\
\hline Current Account & 1,908 & 9,06 & 3,44 & 16,8 \\
\hline Interest Rate & 5 & 3,25 & 8,75 & 3,5 \\
\hline GDP & 2344536 & 308078 & 2552217 & 10206201 \\
\hline \multicolumn{5}{|c|}{ Min Value } \\
\hline Stock Price & 1825,090 & 872,55 & 1285,48 & 431,5 \\
\hline Exchange Rate & 40,6814 & 2,9868 & 8528,203 & 29,0646 \\
\hline Inflation & 0 & $-2,48$ & $-0,45$ & $-4,378$ \\
\hline Current Account & -1.3 & 0,5 & $-10,13$ & -6 \\
\hline Interest Rate & 3 & 2 & 4,25 & 1,25 \\
\hline GDP & 0 & 178543,5 & 0 & 7657100 \\
\hline
\end{tabular}

Characteristic

\begin{tabular}{|c|c|c|c|c|}
\hline Stock Exchange & $\begin{array}{l}\text { Philippine } \\
\text { SE }\end{array}$ & Bursa Malaysia & $\begin{array}{c}\text { Indonesia } \\
\text { SE }\end{array}$ & $\begin{array}{c}\text { SE of } \\
\text { Thailand }\end{array}$ \\
\hline Name index & PSEi & KLCI & IHSG & SETI \\
\hline Number Listed Companies & 265 & 903 & 537 & 656 \\
\hline $\begin{array}{l}\text { Total Volume Traded } 2016 \\
\text { (billion USD) } \\
\text { Market Capitalisation } 2016 \\
\text { (billion USD) }\end{array}$ & 240 & 106 & 426 & 329 \\
\hline $\begin{array}{l}\text { Clasification by IMF } \\
\text {-Emerging Market \& }\end{array}$ & & & & \\
\hline Developing Economies & yes & yes & yes & yes \\
\hline -Emerging \& Developing ASIA & yes & yes & yes & yes \\
\hline
\end{tabular}

Note: processed by author. 
To test the stationarity of variables in this paper, we will use the Phillips-Perron (PP) test with the null hypothesis non stationary variable or have the unit root and the Kwiatkowski-PhillipsSchmidt-Shin (KPSS) test. We do not explain the unit root test methodology in this paper to compress the discussion. By using the Akaike's Information Criteria (AIC), table 2 presents the results of PP unit root test and KPSS between stock price and macroeconomic variables. From table 2 it was found that from four ASEAN-4 countries, variables stationary at different degrees i.e at I (0) and I (1), no variable was found to be integrated in I (2). This means that we can apply the ARDL approach to examine the effect of stock prices and macroeconomic variables.

Table 2. ASEAN-4 Unit Root Test

\begin{tabular}{|c|c|c|c|c|c|c|c|c|c|}
\hline \multirow{3}{*}{$\begin{array}{c}\text { Variable } \\
\text { SP }\end{array}$} & \multicolumn{4}{|c|}{ PP Test } & \multicolumn{4}{|c|}{ KPPS Test } & \multirow{2}{*}{ Countries } \\
\hline & \multirow{2}{*}{\begin{tabular}{|c|} 
Level \\
$-1,4539$
\end{tabular}} & \multicolumn{3}{|c|}{$1^{\text {st }}$ diff } & \multirow{2}{*}{$\begin{array}{c}\text { Level } \\
1,1409\end{array}$} & \multicolumn{3}{|c|}{$1^{\text {st }}$ diff } & \\
\hline & & (1) & $-9,7774 *$ & (1) & & (9) & $0,1057^{* * *}$ & (1) & \multirow{6}{*}{ Philippines } \\
\hline ExR & -0.5343 & (6) & $-8,2151^{*}$ & (13) & $0,4494^{* *}$ & (9) & $0,4633^{*}$ & (6) & \\
\hline Inf & $-3,0483^{* *}$ & (3) & $-6,4053^{*}$ & $(3)$ & 0,7821 & (8) & $0,1318^{* * *}$ & (3) & \\
\hline $\mathrm{CuA}$ & $-1,8569$ & (7) & $-7,6139 *$ & (13) & $0,3182^{* * *}$ & (7) & $0,298^{* * *}$ & (12) & \\
\hline InR & $-2,2092$ & (5) & $-9,4537^{*}$ & $(4)$ & 0,7711 & (8) & $0,0892^{* * *}$ & (5) & \\
\hline GDP & $-4,8868 *$ & $(0)$ & $-9,5986^{*}$ & (8) & 1,3069 & (8) & $0,257^{* * *}$ & (8) & \\
\hline $\mathbf{S P}$ & $-3,8362^{*}$ & (11) & $-10,5922^{*}$ & $(0)$ & 0,9424 & (8) & $0,5495^{*}$ & (2) & \multirow{6}{*}{ Malaysia } \\
\hline ExR & $-0,6339$ & (3) & $-6,9781^{*}$ & (2) & 0,7556 & (9) & $0,2753^{* * *}$ & (3) & \\
\hline Inf & $-3,0611^{* *}$ & (2) & $-6,4509 *$ & (4) & $0,3788^{* *}$ & (7) & $0,1028^{* * *}$ & (0) & \\
\hline $\mathrm{CuA}$ & $-1,5001$ & $(0)$ & $-4,0247^{*}$ & (9) & 1,0664 & (8) & $0,0952^{* * *}$ & (0) & \\
\hline InR & $-1,3725$ & (5) & $-12,5624^{*}$ & $(6)$ & 0,7639 & (9) & $0,1265^{* * *}$ & (5) & \\
\hline GDP & 0,3787 & (5) & $-5,0594^{*}$ & (11) & 1,1974 & (9) & $0,0815^{* * *}$ & (5) & \\
\hline $\mathbf{S P}$ & $-1,8407$ & (4) & $-9,6241^{*}$ & (2) & 1,1871 & (8) & $0,1682^{* * *}$ & (2) & \multirow{6}{*}{ Indonesia } \\
\hline ExR & $-0,2664$ & (5) & $-8,255^{*}$ & (5) & 0,9447 & (9) & $0,3763^{* *}$ & (5) & \\
\hline Inf & $-6,8532 *$ & (37) & $-19,0946^{*}$ & (20) & $0,1563^{* * *}$ & (39) & $0,0913^{* * *}$ & (20) & \\
\hline CuA & $-1,4355$ & $(4)$ & $-4,6058^{*}$ & (29) & '0,6639* & (9) & $0,094^{* * *}$ & (5) & \\
\hline InR & $-1,6859$ & (6) & $-6,5141^{*}$ & $(1)$ & $0,2572^{* * *}$ & (8) & $0,1846^{* * *}$ & (6) & \\
\hline GDP & $-5,6497^{*}$ & (6) & $-10,2541^{*}$ & $(0)$ & 1,2917 & (8) & $0,3192^{* * *}$ & $(0)$ & \\
\hline $\mathbf{S P}$ & $-1,8604$ & (1) & $-9,0641^{*}$ & (1) & 1,049 & (9) & $0,1632^{* * *}$ & (1) & \multirow{6}{*}{ Thailand } \\
\hline ExR & $-1,6063$ & (2) & $-6,3421^{*}$ & (7) & $0,4326^{* *}$ & (9) & $0,2601^{* * *}$ & (1) & \\
\hline Inf & $-1,9317$ & (2) & $-8,4112^{*}$ & $(0)$ & 0,7338* & (8) & $0,0555^{* * *}$ & (1) & \\
\hline $\mathrm{CuA}$ & $-1,3544$ & (7) & $-4,2908^{*}$ & (21) & 0,9343 & (8) & $0.1070^{* * *}$ & (9) & \\
\hline InR & $-1,2912$ & (6) & $-8,8720^{*}$ & (4) & $0,6679^{*}$ & (9) & $0,1194^{* * *}$ & (6) & \\
\hline GDP & 0,3938 & (8) & $-2,9817^{* *}$ & (3) & 1,1694 & (9) & $0,0897^{* * *}$ & (8) & \\
\hline
\end{tabular}

Note: * **, and ${ }^{* * *}$ indicates significant at 1\%, 5\%, and 10\% level. The parenthesis are a lag length based on Akaike's Information Criteria (AIC).

\section{The ASEAN-4 Cointegration Test}

After the unit root test performed, the bound cointegration test of ARDL is applied to see the cointegration between stock prices and macroeconomic variables. One of the advantages of ARDL is that it can be applied to equations where there are different degrees of integration between the variables. Another requirement is ARDL can not be performed if there are stationary variables on I (2). Cointegration test results can be seen in table 3 with the dependent variable is the stock price (SP). In this test, As in the unit root test, lag length for depedent variables and regressors we choose by using AIC criteria for Philippines, Malaysia, Indonesia, and Thailand. To avoid the problem of autocorrelation, for Thailand we use the rule oft humbs lags 10. Brandt and Williams (2007) stated that for the rule of thumbs, the 
autoregressive model must include enough lag to capture the cyclical phenomena in the data. For monthly and quarterly data there are usually monthly cycles that carried over from year to year and month to month, so to strengthen the estimates it should be possible used lag 8 or 10 to solve the problem. The bound test concludes there is the long-term cointegration of four ASEAN-4 countries where the F-statistic value is greater than the critical value of upper bounds I (1) of Pesaran, Shin, and Smith (2001).

Table 3. Bounds Test of Cointegration ASEAN-4

\begin{tabular}{|c|c|c|c|c|c|c|c|c|}
\hline Model: & \multicolumn{4}{|c|}{$\begin{array}{c}\text { FSP(SP|ExR, Inf, CuA, InR, } \\
\text { GDP)Philippines }\end{array}$} & \multicolumn{4}{|c|}{$\begin{array}{l}\text { FSP(SP|ExR, Inf, CuA, InR, } \\
\text { GDP) }\end{array}$} \\
\hline $\begin{array}{l}\text { F-Statistics } \\
\text { (Bound Test) }\end{array}$ & \multicolumn{4}{|c|}{$3.1458^{*}$} & \multicolumn{4}{|c|}{$4.2154^{*}$} \\
\hline Critical Values & $1 \%$ & $2.50 \%$ & $5 \%$ & $10 \%$ & $1 \%$ & $2.50 \%$ & $5 \%$ & $10 \%$ \\
\hline $\begin{array}{l}\text { Upper Bounds } \\
\text { I(1) } \\
\text { Lower Bounds }\end{array}$ & 3.06 & 2.7 & 2.39 & 2.08 & 4.15 & 3.73 & 3.38 & 3 \\
\hline $\mathrm{I}(0)$ & 4.15 & 3.73 & 3.38 & 3 & 3.06 & 2.7 & 2.39 & 2.08 \\
\hline $\mathrm{k}$ & \multicolumn{4}{|c|}{5} & \multicolumn{4}{|c|}{5} \\
\hline Lag length & \multicolumn{4}{|c|}{$(5,5)$} & \multicolumn{4}{|c|}{$(8,8)$} \\
\hline $\mathrm{R}^{2}$ & \multicolumn{4}{|c|}{0.3658} & \multicolumn{4}{|c|}{0.3385} \\
\hline Adj. $\mathrm{R}^{2}$ & \multicolumn{4}{|c|}{0.2625} & \multicolumn{4}{|c|}{0.2333} \\
\hline F-Statistics & \multicolumn{4}{|c|}{3.5428} & \multicolumn{4}{|c|}{3.2171} \\
\hline
\end{tabular}

\begin{tabular}{|c|c|c|c|c|c|c|c|c|}
\hline Model: & \multicolumn{4}{|c|}{$\begin{array}{c}\text { FSP(SP|ExR, Inf, CuA, InR, } \\
\text { GDP) Indonesia }\end{array}$} & \multicolumn{4}{|c|}{$\begin{array}{c}\text { FSP(SP|ExR, Inf, CuA, InR, } \\
\text { GDP) Thailand }\end{array}$} \\
\hline $\begin{array}{l}\text { F-Statistics } \\
\text { (Bound Test) }\end{array}$ & \multicolumn{4}{|c|}{$4.1062^{*}$} & \multicolumn{4}{|c|}{$3.7522^{*}$} \\
\hline Critical Values & $1 \%$ & $2.50 \%$ & $5 \%$ & $10 \%$ & $1 \%$ & $2.50 \%$ & $5 \%$ & $10 \%$ \\
\hline $\begin{array}{l}\text { Upper Bounds } \\
\text { I(0) } \\
\text { Lower Bounds }\end{array}$ & 3.06 & 2.7 & 2.39 & 2.08 & 3.06 & 2.7 & 2.39 & 2.08 \\
\hline $\mathrm{I}(1)$ & 4.15 & 3.73 & 3.38 & 3 & 4.15 & 3.73 & 3.38 & 3 \\
\hline $\mathrm{k}$ & \multicolumn{4}{|c|}{5} & \multicolumn{4}{|c|}{5} \\
\hline Lag length & \multicolumn{4}{|c|}{$(6,6)$} & \multicolumn{4}{|c|}{$(10,10)$} \\
\hline $\mathrm{R}^{2}$ & \multicolumn{4}{|c|}{0.3317} & \multicolumn{4}{|c|}{0.5915} \\
\hline Adj. $R^{2}$ & \multicolumn{4}{|c|}{0.2341} & \multicolumn{4}{|c|}{0.4646} \\
\hline F-Statistics & \multicolumn{4}{|c|}{3.3982} & \multicolumn{4}{|c|}{4.6591} \\
\hline
\end{tabular}

Note: * indicates significant at $1 \%$ level.

For the short term it is concluded from the four ASEAN-4 countries there is a short-term cointegration where $\mathrm{ECT}_{\mathrm{t}-1}$ values are negative and significant at $1 \%$ level. This indicates that the presence of disequilibrium at current time will be corrected in the next month for each ASEAN-4 country. For Philippines, 23.24\% disequilibrium at current time will be corrected in the next month.While Malaysia, Indonesia, and Thailand are 18.54\%, 14.12\%, and 11.16\% respectively. Table 4 is an ARDL with error correction model and long-term estimation of ASEAN-4. From the long-term equation, it is found that exchange rate and GDP have a significant effect on stock prices in all ASEAN-4 countries. While other variables have no effect, except current account for Malaysia. 
Table 4. ARDL Cointegration for ASEAN-4

\begin{tabular}{|c|c|c|c|c|}
\hline \multicolumn{5}{|c|}{ Estimation 1: ARDL with ECM } \\
\hline \multirow{2}{*}{ Variable } & \multicolumn{4}{|c|}{ Dependent Variable $=\mathrm{SP}$} \\
\hline & Philippines & Malaysia & Indonesia & Thailand \\
\hline \multirow[t]{2}{*}{$\Delta$ SPt-1 } & -0.0902 & -0.2018 & & -0.2997 \\
\hline & $(-0.8994)$ & $(-1.8338)^{* * *}$ & & $(-2.7755)^{*}$ \\
\hline \multirow[t]{2}{*}{$\Delta \mathrm{SPt}-2$} & 0.0576 & & & -0.1941 \\
\hline & $(0.6132)$ & & & $(-2.1708)^{* *}$ \\
\hline \multirow[t]{2}{*}{$\Delta \mathrm{SPt}-3$} & 0.194 & & & \\
\hline & $(2.0419)^{* *}$ & & & \\
\hline \multirow[t]{2}{*}{$\Delta \mathrm{ExR}$} & -210.9369 & -242.0652 & -0.3614 & -86.6304 \\
\hline & $(-4.9936)^{*}$ & $(-4.0786)^{*}$ & $(-4.1896)^{*}$ & $(-6.8881)^{*}$ \\
\hline \multirow[t]{2}{*}{$\Delta$ ExRt-1 } & & 73.9366 & & \\
\hline & & $(0.7863)$ & & \\
\hline \multirow[t]{2}{*}{$\Delta$ ExRt-2 } & & -14.9266 & & \\
\hline & & $(-0.1613)$ & & \\
\hline \multirow[t]{2}{*}{$\Delta$ ExRt-3 } & & -54.4877 & & \\
\hline & & $(-0.6106)$ & & \\
\hline \multirow[t]{2}{*}{$\Delta$ ExRt-4 } & & -104.9151 & & \\
\hline & & $(1.898)^{* * *}$ & & \\
\hline \multirow[t]{2}{*}{$\Delta \operatorname{Inf}$} & -102.1008 & 0.7396 & 19.1263 & 15.9805 \\
\hline & $(1.7162)^{* * *}$ & $(0.1762)$ & $(0.6023)$ & $(3.0061)^{* *}$ \\
\hline \multirow[t]{2}{*}{$\Delta \operatorname{Inf}$} & -13.0216 & & -54.7778 & \\
\hline & $(-0.136)$ & & $(-1.5084)$ & \\
\hline \multirow[t]{2}{*}{$\Delta \operatorname{Inf}$} & 42.3244 & & -83.6278 & \\
\hline & $(0.4637)$ & & $(-2.2902)^{*}$ & \\
\hline \multirow[t]{2}{*}{$\Delta \operatorname{Inf}$} & -73.2492 & & 116.3183 & \\
\hline & $(-1.3056)$ & & $(3.3794)^{*}$ & \\
\hline \multirow[t]{2}{*}{$\Delta \operatorname{Inf}$} & & & -56.1215 & \\
\hline & & & $(-1.6123)$ & \\
\hline \multirow[t]{2}{*}{$\Delta \mathrm{CuA}$} & 36.8899 & -4.7725 & -5.6435 & -4.6962 \\
\hline & $(0.8188)$ & $(-1.6409)$ & $(-0.9084)$ & $(-1.6195)$ \\
\hline \multirow[t]{2}{*}{$\Delta \mathrm{InR}$} & -35.8612 & -33.3957 & 9.9298 & 31.6267 \\
\hline & $(-0.4843)$ & $(-1.4274)$ & $(0.4386)$ & $(0.5118)$ \\
\hline \multirow[t]{2}{*}{$\Delta$ InRt-1 } & & & & -40.3742 \\
\hline & & & & $(-0.4197)$ \\
\hline \multirow[t]{2}{*}{$\Delta \mathrm{InRt}-2$} & & & & -94.7006 \\
\hline & & & & $(-1.4571)$ \\
\hline \multirow[t]{2}{*}{$\Delta \mathrm{GDP}$} & 0.0006 & 0.0014 & 0.0001 & 0.00004 \\
\hline & $(0.9708)$ & $(0.7562)$ & $(0.0425)$ & $(0.0887)$ \\
\hline \multirow[t]{2}{*}{$\Delta \mathrm{GDPt}-1$} & -0.0014 & -0.0035 & 0.0044 & -0.001 \\
\hline & $(-2.1806)^{* *}$ & $(-1.7168)^{* * *}$ & $(1.8853)^{* * *}$ & $(-0.8856)$ \\
\hline \multirow[t]{2}{*}{$\Delta \mathrm{GDPt}-2$} & -0.00009 & & -0.0038 & 0.0002 \\
\hline & $(-0.5389)$ & & $(-3.0091)^{*}$ & $(0.2289)$ \\
\hline$\Delta \mathrm{GDPt}-3$ & & & -0.0001 & 0.00002 \\
\hline & & & $(-1.1593)$ & $(0.0172)$ \\
\hline$\Delta \mathrm{GDPt}-4$ & & & 0.0002 & -0.0009 \\
\hline & & & $(2.1561)^{* *}$ & $(-0.8004)$ \\
\hline
\end{tabular}




\begin{tabular}{|c|c|c|c|c|}
\hline$\Delta \mathrm{GDPt}-5$ & & & & $\begin{array}{c}0.0009 \\
(0.8071)\end{array}$ \\
\hline$\Delta$ GDPt-6 & & & & $\begin{array}{c}-0.001 \\
(-0.9233)\end{array}$ \\
\hline$\Delta \mathrm{GDPt}-7$ & & & & $\begin{array}{c}0.0014 \\
(1.3446)\end{array}$ \\
\hline$\Delta \mathrm{GDPt}-8$ & & & & $\begin{array}{c}0.0005 \\
(0.4872)\end{array}$ \\
\hline$\Delta$ GDPt-9 & & & & $\begin{array}{c}-0.001 \\
(-2.0751)^{* *}\end{array}$ \\
\hline ECTt-1 & $\begin{array}{c}-0.2324 \\
(-3.6343)^{*}\end{array}$ & $\begin{array}{c}-0.1854 \\
(-2.9064)^{*}\end{array}$ & $\begin{array}{c}-0.1412 \\
(-2.5942)^{*}\end{array}$ & $\begin{array}{c}-0.1168 \\
(-1.6708)^{* * *}\end{array}$ \\
\hline \multicolumn{5}{|c|}{ Estimation 2: Long Run Coeficcients } \\
\hline & Philippines & Malaysia & Indonesia & Thailand \\
\hline $\mathrm{C}$ & $\begin{array}{c}2706.4962 \\
{[0.3427]}\end{array}$ & $\begin{array}{c}1955.7479 \\
{[0.0007]^{*}}\end{array}$ & $\begin{array}{c}3638.6282 \\
{[0.094]^{* * *}}\end{array}$ & $\begin{array}{c}2089.0586 \\
{[0.1935]}\end{array}$ \\
\hline ExR & $\begin{array}{l}223.2993 \\
{[0.0000]^{*}}\end{array}$ & $\begin{array}{c}-408.0369 \\
{[0.0000]^{*}}\end{array}$ & $\begin{array}{c}-0.4628 \\
{[0.0195]^{*}}\end{array}$ & $\begin{array}{c}-108.7932 \\
{[0.0623]^{* * *}}\end{array}$ \\
\hline Inf & $\begin{array}{c}76.4536 \\
{[0.5237]}\end{array}$ & $\begin{array}{c}3.9908 \\
{[0.8582]}\end{array}$ & $\begin{array}{c}172.5932 \\
{[0.7909]}\end{array}$ & $\begin{array}{c}136.7661 \\
{[0.1874]}\end{array}$ \\
\hline $\mathrm{CuA}$ & $\begin{array}{c}158.7245 \\
{[0.3829]}\end{array}$ & $\begin{array}{c}-25.7486 \\
{[0.0913]^{* * *}}\end{array}$ & $\begin{array}{c}-39.9732 \\
{[0.3657]}\end{array}$ & $\begin{array}{c}16.563 \\
{[0.4376]}\end{array}$ \\
\hline InR & $\begin{array}{c}-154.2986 \\
{[0.6348]}\end{array}$ & $\begin{array}{c}-180.1752 \\
{[0.2073]}\end{array}$ & $\begin{array}{l}70.3327 \\
{[0.6537]}\end{array}$ & $\begin{array}{c}-104.1137 \\
{[0.4288]}\end{array}$ \\
\hline GDP & $\begin{array}{c}0.0081 \\
{[0.0000]^{*}}\end{array}$ & $\begin{array}{c}0.0074 \\
{[0.0003]^{*}}\end{array}$ & $\begin{array}{c}0.0064 \\
{[0.0002]^{*}}\end{array}$ & $\begin{array}{c}0.0008 \\
{[0.0063]^{*}}\end{array}$ \\
\hline
\end{tabular}

Note: *, **, and *** indicates significant at $1 \%, 5 \%$, and $10 \%$ level.

Round parenthesis show t-statistics, square parenthesis indicates prob. Value.

\section{Stability and Diagnostic Check}

Diagnostic tests for serial correlation, heteroskedasticity, and normality tests can be seen in Table 5 where we conclude there is no time series problem in our estimation. Ramsey Reset test also applied with the null hypothesis the model has been specified correctly. For four countries, we accept the null hypothesis which concludes that there is no misspecified problem in our equation. 
Table 5. Stability Diagnostic Check for ASEAN-4

\begin{tabular}{ccccc}
\hline \hline Countries & $\mathbf{B G}_{\mathbf{s c}}$ & $\mathbf{B P G}_{\mathbf{h t}}$ & $\mathbf{J B}_{\mathbf{n t}}$ & $\begin{array}{c}\text { Ramsey Reset } \\
\text { Test }\end{array}$ \\
\hline Philippines & $7.6934(0.174)$ & 15.6381 & 2.9969 & $0.5496)$ \\
Malaysia & $6.0288(0.644)$ & $(0.0595)$ & $(0.1438)$ & $0.8992(0.3456)$ \\
& & 16.9958 & 1.8559 & \\
Indonesia & $5.6307(0.4658)$ & $(0.4547)$ & $(0.3954)$ & $2.9277(0.0591)$ \\
& 14.3844 & 31.8092 & 1.0848 & \\
Thailand & $(0.1562)$ & $(0.1042)$ & $(0.5814)$ & $0.1293(0.7202)$ \\
\hline \hline
\end{tabular}

Note: $\mathrm{BG}_{\mathrm{sc}}, \mathrm{BPG}_{\mathrm{ht}}$, and $\mathrm{JB}_{\mathrm{nt}}$, is a Breusch-Pagan serial correlation, Breusch-Pagan-Godfrey heteroskedasticity, and Jarque-Bera normality test. Round parenthesis show prob. Value.

The interesting result from the long-term findings from the ASEAN-4 countries we found a similar failure pattern on the inflation, interest rate, and current account on average in three of the four ASEAN-4. So to corroborate our previous results, in this paper we will estimate the influence of each variable of inflation, current account, and interest rate in "one for one" sceme against stock price by bivariate analysis. For efficiency, the unit root test and cointegration results are not shown in this paper. From the cointegration test of Engle-Granger and PhillipsOuliaris concluded that there is no cointegration in three ASEAN-4 countries namely Philippines, Indonesia, and Thailand. While for Malaysia because it is concluded that there is cointegration so we will use ECM compared to autoregressive bivariate (BVAR) in Phillippines, Indonesia, and Thailand. We can write BVAR equations for each country as follows:

$$
\begin{aligned}
& x_{t}=S_{0}+\sum_{j=1}^{p} g_{j} x_{t-j}+\sum_{j=1}^{p} h_{j} y_{t-j}+u_{x t \ldots \ldots \ldots} \\
& y_{t}=\kappa_{0}+\sum_{j=1}^{p} i_{j} x_{t-j}+\sum_{j=1}^{p} j_{j} y_{t-j}+u_{y t \ldots \ldots \ldots . .}
\end{aligned}
$$

Table 6 is a variance decomposition with the stock price is dependent variable. From the result of variance decomposition we can know whether the contribution of regressor is too small or not to influenced the formation of stock price. 
Table 6. Contribution Inf, CuA, and InR on SP

\begin{tabular}{|c|c|c|c|c|c|c|c|c|c|c|c|}
\hline \multirow{3}{*}{ Period } & \multicolumn{11}{|c|}{ Variance Decomposition of SP } \\
\hline & \multicolumn{3}{|c|}{ Philippines } & \multicolumn{2}{|c|}{ Malaysia } & \multicolumn{3}{|c|}{ Indonesia } & \multicolumn{3}{|c|}{ Thailand } \\
\hline & Inf & $\mathrm{CuA}$ & InR & Inf & InR & Inf & CuA & InR & Inf & CuA & InR \\
\hline 1 & 0.0000 & 0.0000 & 0.0000 & 0.0000 & 0.0000 & 0.0000 & 0.0000 & 0.0000 & 0.0000 & 0.0000 & 0.0000 \\
\hline 2 & 0.0002 & 0.2189 & 0.0305 & 0.1493 & 0.2042 & 3.13 & 0.1498 & 0.5203 & 0.0001 & 0.0042 & 1.1454 \\
\hline 3 & 0.0556 & 0.5117 & 0.0965 & 0.1166 & 0.1453 & 4.599 & 0.1029 & 1.1194 & 0.0004 & 0.1086 & 1.4388 \\
\hline 4 & 0.2867 & 0.7785 & 0.193 & 0.8502 & 0.1403 & 3.2973 & 0.0785 & 1.6873 & 0.0007 & 0.4985 & 1.7909 \\
\hline 5 & 0.7209 & 0.9956 & 0.3153 & 2.9157 & 0.2263 & 4.9835 & 0.0662 & 2.2139 & 0.0012 & 0.1307 & 1.7824 \\
\hline 6 & 1.3127 & 1.1653 & 0.4594 & 5.9439 & 0.4114 & 7.9097 & 0.0725 & 2.7095 & 0.0017 & 1.6033 & 1.7757 \\
\hline 7 & 1.9915 & 1.2964 & 0.6215 & 9.259 & 0.6942 & 9.1798 & 0.1288 & 3.1843 & 0.0024 & 2.2114 & 1.9205 \\
\hline 8 & 2.6924 & 1.3982 & 0.7984 & 12.3671 & 1.0696 & 10.1335 & 0.1956 & 3.6459 & 0.003 & 2.9056 & 2.0119 \\
\hline 9 & 3.3692 & 1.4782 & 0.9872 & 15.0431 & 1.5308 & 10.2661 & 0.2584 & 4.0992 & 0.0038 & 3.5795 & 2.0858 \\
\hline 10 & 3.9949 & 1.542 & 0.1853 & 17.2472 & 2.0699 & 10.2925 & 0.2749 & 4.5472 & 0.0045 & 4.0639 & 2.0264 \\
\hline \multicolumn{12}{|c|}{ Diagnostic Test } \\
\hline $\mathbf{p}$ & 2 & 2 & 1 & 2 & 1 & 6 & 6 & 2 & 1 & 7 & 6 \\
\hline \multirow[t]{7}{*}{$\chi^{2} \mathrm{sc}$} & 0.9618 & 0.1169 & 0.767 & 0.5222 & 0.1072 & 0.9946 & 0.6433 & 0.0384 & 0.1396 & 0.8548 & 0.2308 \\
\hline & 0.7066 & 0.4892 & - & 0.6922 & & 0.7433 & 0.7277 & 0.2384 & & 0.4356 & 0.6858 \\
\hline & & & & & & 0.1349 & 0.0783 & & & 0.0000 & 0.6938 \\
\hline & & & & & & 0.3732 & 0.2126 & & & 0.0786 & 0.0991 \\
\hline & & & & & & 0.5236 & 0.4205 & & & 0.2917 & 0.6713 \\
\hline & & & & & & 0.3903 & 0.0069 & & & 0.0019 & 0.1727 \\
\hline & & & & & & & & & & 0.4272 & \\
\hline$\chi^{2} \mathrm{ht}$ & - & 0.1529 & 0.4695 & - & 0.9635 & 0.3628 & 0.4599 & - & 0.0258 & 0.2985 & - \\
\hline
\end{tabular}

Note: $\mathrm{p}$ is lag length, $\chi^{2}$ sc is serial correlation test, $\chi^{2}$ ht is a heteroskedasticity test based on bivariate analysis "one for one" regressors against SP.

From table 6 it can be concluded even at the end of the tenth period, the contribution of regressors has increased but their amounts is very small to influence the formation of stock price in ASEAN-4 countries. This finding strengthen our previous result. Finally, the test of cusum and cusumq we applied to test the stability of the model that we propose. It is seen that from ASEAN-4 model, we conclude that our equation has fulfilled the diagnostic test and also the stability test, where cusum and cusumq are within the $5 \%$ significance limit. 


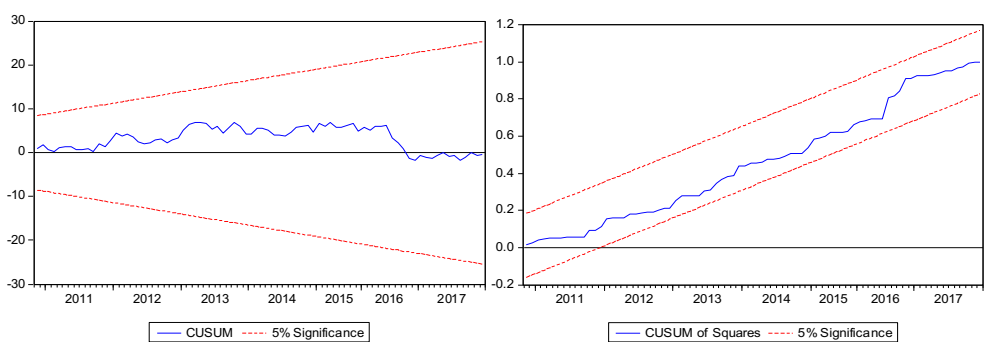

(a) Philippines Cusum dan Cusumq
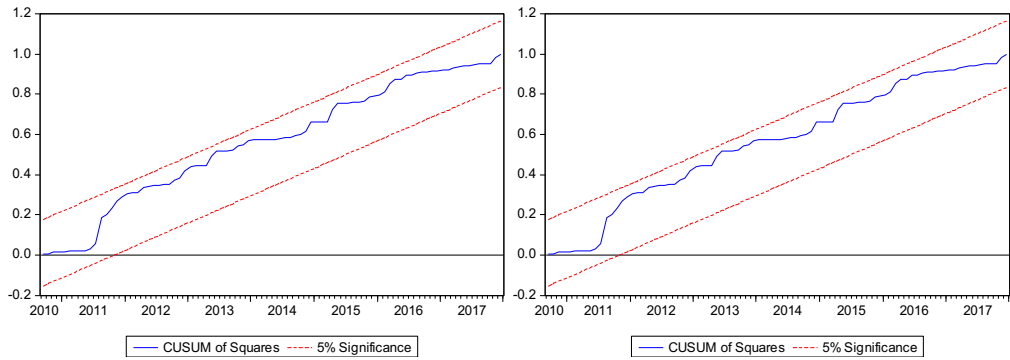

(b) Malaysia Cusum dan Cusumq

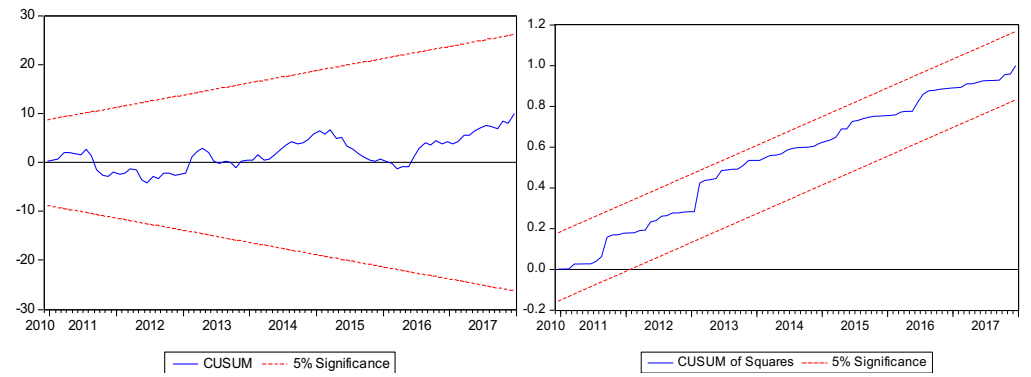

(c) Indonesia Cusum dan Cusumq

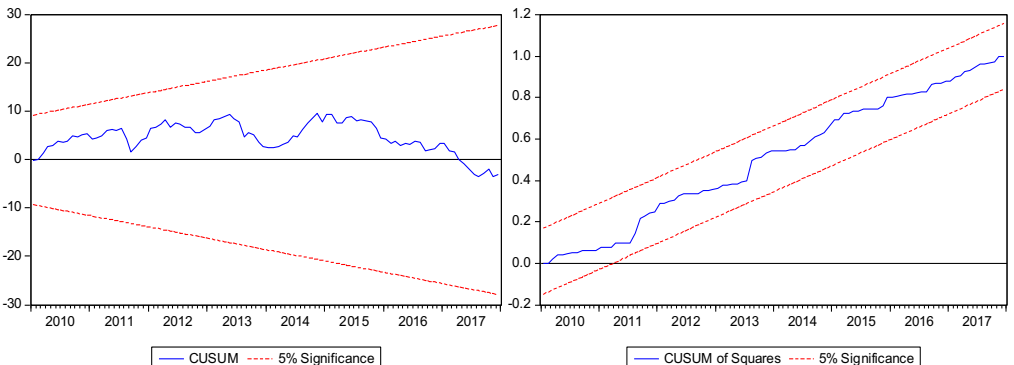

(d) Thailand Cusum dan Cusumq

\section{CONCLUSION AND SUGGESTION}

The effect of macroeconomic variables on stock prices is much debated. Research on this issue in countries that using the IMF classification is still limited. Given the similarity of macroeconomic characteristic in ASEAN-4 countries namely Philippines, Malaysia, Indonesia, and Thailand also resulted similar findings around causality the macroeconomic variable and stock prices. In addition to short and long-term cointegration for all ASEAN-4 countries, exchange rates and GDP in the long run strongly indicate a significant effect on stock prices.While other macroeconomic variables such as inflation, current accounts and GDP do not show strong evidence to influence the stock price formation. By applying the "one to one" scheme, inflation, current accounts, and interest rates concluded play a small role among other macroeconomic variables. The very small contribution is an initial indication of the failure of some macroeconomic variables in explaining the force of the capital market. The important question now: do we excessively to modeled the capital markets through many macroeconomic forces?, or do we have to go back to CAPM?. 


\section{Reference}

Bahloul, Slah, Mourad Mroua, and Nader Naifar, 2017. "The Impact of Macroeconomic and Conventional Stock Market Variables on Islamic Index Return under Regime Switching”, Borsa Istanbul Review, 17-1: 62-74.

Belgacem, Aymen, 2013. "Explaining the Stock Market's Reaction to Macroeconomic Announcements", retrieved from https://halshs.archieve-ouvertes.fr/halshs-01064891, accessed on 28 April 2018.

Brandt, Patrick T., and John T., Williams, 2007. Multiple Time Series Models: Quantitative Applications in The Social Science, Sage Publication.Inc, California.

Chen, Nai-Fu, Richard Roll, and Stephen A. Ross, 1986. "Economic Force and the Stock Market", The Journal of Business, 59 (3): 383-403.

Huberman, Gur, and Zhenyu Wang, 2015. "Arbitrage Pricing Theory”, The New Palgrave Dictionary of Economics, 2nd Edition, S. N. Durlauf and L. E. Blume (eds), Palgrave Macmillan.

Ouma, Wycliffe Nduga, and Peter Muriu, 2014. "The Impact of Macroeconomic Variables on Stock Market Returns in Kenya", International Journalof Business and Commerce, 3 (11): 1-31.

Parsva, Parham, and Chor Foon Tang, 2017. "A Note on Interaction between Stock Price and Exchange Rate in Middle-East Economies”, Economic Research-Ekonomska Istrazivanja, 30 (1): 838-844.

Patel, Samveg, 2012. "The Effect of Macroeconomic Determinants on the Performance of the Indian Stock Market", NMIMS Management Review, 22: 117-127.

Pesaran, M. H, Yongcheol Shin, and Richard J. Smith, 2001. "Bounds Testing Approaches to the Analysis of Level Relationship", Journal of Applied Econometrics, 16: 289-326.

Pilinkus, Donatas, 2011. "Macroeconomic Indicators and Their Impact on Stock Market Performance in the Short and Long Run: The Case of the Baltic States". Technological and Economic Development of Economy, 16 (2): 291 304.

Quadir, Muhammed Monjurul, 2012. "The Effect of Macroeconomic Variables on Stock Returns on Dhaka Stock Exchange", International Journal of Economics and Financial Issues, 2 (4): 480-487.

Rahman, Md. Lutfur, and Jashim Uddin, 2009. "Dynamic Relationship between Stock Price and Exchange Rates: Evidence from Three South Asian Countries”, International Business Research, 2 (2): 167-174.

Rasiah, Devinaga, and PeongKwee Kim, 2011. "The Effectiveness of Arbitrage Pricing Model in Modern Financial Theory", International Journal Economics and Research, 2(3): 125-135.

Reza, Faizal, Adisthy Shabrina M., and Danna Solihin, 2018. "Is Arbitrage Pricing Theory Is a Fairy Tale ?: The Evidence From Indonesia with Ordinary Least Square Estimation", Research Journal of Accounting and Business Management, 2 (1): 18-31.

Sharma, Gagan Deep, and Mandeep Mahendru, 2010. "Impact of Macro-Economic Variableson Stock Price in India”, Global Journal of Management and Business Research, 10 (7): 19-26.

Umoru, David, and Mike O. Asekome, 2013. "Stock Price and Exchange Rate Variability in Nigeria Econometric Analysis of the Evidence", European Scientific Journal, 9 (25): 261-285.

Wickremasinghe, G., B., 2006. "Macroeconomic Forces and Stock Price: Some Empirical Evidence from an Emerging Stock Market”, Accountingand Finance Working Paper 06/14.

Yau, Hwey-Yun, and Chien-Chung Nieh, 2008. "Testing for Cointegration with Threshold Effect between Stock Price and Exchange Rates in Japan and Taiwan", Japan and the World Economy, 21: 292-300.

Zubair, Abdulrasheed, 2013. "Causal Relationship between Stock Market Index and Exchange Rate: Evidence from Nigeria”, CBN Journal of Applied Statistics, 4 (2): 87-110. 